\title{
Extracting certainty from uncertainty: regret bounded by variation in costs
}

\author{
Elad Hazan • Satyen Kale
}

Received: 15 March 2009 / Accepted: 1 November 2009 / Published online: 29 April 2010

(C) The Author(s) 2010

\begin{abstract}
Prediction from expert advice is a fundamental problem in machine learning. A major pillar of the field is the existence of learning algorithms whose average loss approaches that of the best expert in hindsight (in other words, whose average regret approaches zero). Traditionally the regret of online algorithms was bounded in terms of the number of prediction rounds.

Cesa-Bianchi, Mansour and Stoltz (Mach. Learn. 66(2-3):21-352, 2007) posed the question whether it is be possible to bound the regret of an online algorithm by the variation of the observed costs. In this paper we resolve this question, and prove such bounds in the fully adversarial setting, in two important online learning scenarios: prediction from expert advice, and online linear optimization.
\end{abstract}

Keywords Individual sequences · Prediction with expert advice · Online learning · Regret minimization

\section{Introduction}

A cornerstone of modern machine learning are algorithms for prediction from expert advice. As early as the 1950's (Hannan 1957), in the context of sequential decision making, prediction algorithms were developed which, under fully adversarial cost sequences, attain average cost approaching that of the best expert in hindsight.

Editors: Sham Kakade and Ping Li.

Work done while S. Kale was at Microsoft Research.

E. Hazan $(\varangle)$

IBM Almaden Research Center, 650 Harry Rd, San Jose, CA 95120, USA

e-mail: ehazan@cs.princeton.edu

S. Kale

Yahoo! Research, 4301 Great America Parkway, Santa Clara, CA 95054, USA

e-mail: skale@yahoo-inc.com 
To be more precise, consider a prediction setting in which an online learner has access to $n$ experts. Iteratively, the learner may chose the advice of any expert deterministically or randomly. After choosing a course of action, an adversary reveals the cost of following the advice of the different experts, from which the expected cost of the online learner is derived. The classic results in prediction theory (surveyed in Sect. 1.5) give algorithms which sequentially produce randomized decisions, such that the difference between the (expected) cost of the algorithm and the best expert in hindsight grows like $O(\sqrt{T \log n})$, where $T$ is the number of prediction iterations. This extra additive cost is known as the regret of the online learning algorithm.

However, a priori it is not clear why online learning algorithms should have high regret (growing with the number of iterations) in an unchanging environment. As an extreme example, consider a setting in which there are only two experts. Suppose that the first expert always incurs cost 1 , whereas the second expert always incurs cost $\frac{1}{2}$. One would expect to "figure out" this pattern quickly, and focus on the second expert, thus incurring a total cost that is at most $\frac{T}{2}$ plus at most a constant extra cost (irrespective of the number of rounds $T$ ), thus having only constant regret. However, any straightforward application of previously known analyses of expert learning algorithms only gives a regret bound of $\Theta(\sqrt{T})$ in this simple case (or very simple variations of it).

More generally, the natural bound on the regret of a "good" learning algorithm should depend on variation in the sequence of costs, rather than purely on the number of iterations. If the cost sequence has low variation, we expect our algorithm to be able to perform better.

This intuition has a direct analog in the stochastic setting: here, the sequence of experts' costs are independently sampled from a distribution. In this situation, a natural bound on the rate of convergence to the optimal expert is controlled by the variance of the distribution (low variance should imply faster convergence). This was formalized by Cesa-Bianchi et al. (2007), who assert that "proving such a rate in the fully adversarial setting would be a fundamental result".

In this paper we prove the first such regret bounds on online learning algorithms in two important scenarios: prediction from expert advice, and the more general framework of online linear optimization. Our algorithms have regret bounded by the variation of the cost sequence, in a manner that is made precise in the following sections. Thus, our bounds are tighter than all previous bounds, and hence yield better bounds on the applications of previous bounds (see, for example, the applications in Cesa-Bianchi et al. 2007).

\subsection{Online linear optimization}

Online linear optimization (Kalai and Vempala 2005) is a general framework for online learning which has received much attention recently. In this framework the decision set is an arbitrary bounded, closed, convex set in Euclidean space $K \subseteq \mathbb{R}^{n}$ rather than a fixed set of experts, and the costs are determined by adversarially constructed vectors, $f_{1}, f_{2}, \ldots \in \mathbb{R}^{n}$, such that the cost of point $x \in K$ is given by $f_{t} \cdot x$. The online learner iteratively chooses a point in the convex set $x_{t} \in K$. Then the cost vector $f_{t}$ is revealed and the cost $f_{t} \cdot x_{t}$ is incurred. The performance of online learning algorithms is measured by the regret, which is defined as the difference in the total cost of the sequence of points chosen by the algorithm, viz. $\sum_{t=1}^{T} f_{t} \cdot x_{t}$, and the total cost of the least cost fixed point in hindsight, viz. $\min _{x \in K} \sum_{t=1}^{T} f_{t} \cdot x$.

Several decision problems fit very naturally in this framework. For example, in the online shortest path problem the online learner has to repeatedly choose a path in a given graph from a source node to a destination node. Her cost is the total length of the path according 
to weights which are chosen by an adversary. This problem can be cast as an online linear optimization problem, where the decision space is the set of all distributions over paths in the graph connecting the source to the destination. Even though this set sits in exponential dimensional Euclidean space, by thinking of a distribution over paths as a flow in the graph, it is possible to efficiently represent the decision space as a polytope in $\mathbb{R}^{|E|}$ ( $E$ denotes the set of edges in the given graph), described by $O(|E|)$ constraints, and translate the cost functions to this new domain as well.

The general online linear optimization framework allows for efficient and natural algorithms based on the gradient descent update rule coupled with Euclidean projections (Helmbold et al. 1999; Zinkevich 2003). In this paper, we consider Zinkevich's Lazy Projection algorithm (Zinkevich 2003). This algorithm runs online gradient descent on an auxiliary sequence of points and chooses the projections of these auxiliary points on the convex set in every iteration.

This algorithm was shown to have regret $O(\sqrt{T})$. We improve this regret bound to square-root of the total variation of cost vectors (defined more precisely momentarily). The crucial geometric intuition which allows us to prove regret bounds based on the variation of the cost sequence can be summarized by the following intuitive fact: the distance between successive projections for the Lazy Projection algorithm is directly related to the deviation of the current cost vector from the mean.

We now describe our bounds. Define the variation of the sequence of cost functions to be $\operatorname{VAR}_{T}=\sum_{t=1}^{T}\left\|f_{t}-\mu_{T}^{\star}\right\|^{2}$, where $\mu_{T}^{\star}=\frac{1}{T} \sum_{t=1}^{T} f_{t}$ is the mean of the sequence. Our analysis of the Lazy Projection algorithm yields the following regret bound:

$$
\text { Regret } \leq O\left(\sqrt{\mathrm{VAR}_{T}}\right) .
$$

\subsection{Prediction from expert advice}

Prediction from expert advice can be cast as a special case of online linear optimization: the decision space is the simplex of all distributions on $n$ experts. The expectation operator provides a linear cost function on the simplex via the costs of the experts. Hence, our result for online linear optimization already implies variation bounds for regret in the case of prediction from expert advice.

However, this bound is suboptimal, as it depends on the variation of all experts rather than, say, the maximum variation of a single expert. This issue is familiar to learning theorists: "Euclidean algorithms" such as gradient descent attain performance which relates to the Euclidean norm of the cost functions (or variations in our case). While this Euclidean flavor is optimal in certain cases (for example, when the underlying convex set is the hypercube), for certain convex bodies such as the simplex, better performance can be achieved. The multiplicative update algorithms such as EG (Kivinen and Warmuth 1997) and FPL* (Kalai and Vempala 2005) attain regret which is proportional to $O(R \sqrt{T \log n})$ where $R$ is a bound on the $\ell_{\infty}$ norm of the cost functions.

By analogy with the online linear optimization case, for a sequence of cost vectors $f_{1}, f_{2}, \ldots, f_{T} \in \mathbb{R}^{n}$, where $f_{t}(i)$ is the cost of expert $i$ in the $t^{\text {th }}$ round, we would expect to be able to bound the regret of online linear optimization over the simplex by something like $O\left(\sqrt{\operatorname{VAR}_{T}^{\infty} \log n}\right)$, where

$$
\operatorname{VAR}_{T}^{\infty}=\max _{i \in n}\left\{\sum_{t=1}^{T}\left|f_{t}(i)-\mu_{T}^{\star}(i)\right|^{2}\right\}
$$


is the maximum variation in costs amongst the different experts (as before, $\mu_{T}^{\star}(i)=$ $\frac{1}{T} \sum_{t=1}^{T} f_{t}(i)$ is the mean cost of the $i^{\text {th }}$ expert). In fact, our bound is even stronger,

$$
\operatorname{Regret}(T)=O\left(\sqrt{\mathrm{VAR}_{T}^{\max } \log n}\right) .
$$

Here $\operatorname{VAR}_{T}^{\max } \leq \mathrm{VAR}_{T}^{\infty}$, and is defined to be

$$
\operatorname{VAR}_{T}^{\max }=\max _{t \leq T}\left\{\operatorname{VAR}_{t}\left(\ell_{t}\right)\right\}
$$

where $\operatorname{VAR}_{t}(i)$ is the variation in costs of expert $i$ up to the $t^{\text {th }}$ round, and $\ell_{t}$ is the best expert till the $t^{\text {th }}$ round.

Whereas for the general online linear optimization we analyze the well-known Lazy Projection algorithm and our results are novel by tighter analysis, for the case of prediction from expert advice we need to consider a new algorithm. We can prove that existing variants of the multiplicative weights algorithms do not attain the performance above, and instead consider a different variant of update rule, in which the distribution at time $t$, denoted $x_{t}$ is defined to be

$$
x_{t}(i) \propto \exp \left(-\eta \sum_{\tau=1}^{t-1} f_{\tau}(i)-4 \eta^{2} \sum_{\tau=1}^{t-1}\left(f_{\tau}(i)-\mu_{\tau}(i)\right)^{2}\right),
$$

where $\eta$ is a learning rate parameter and $\mu_{t}=\frac{1}{t} \sum_{\tau=1}^{t-1} f_{\tau}$ is the (approximate) mean cost vector at iteration $t$. That is, the distribution over experts explicitly takes into account the variation in their costs. As far as we know this is a new variant of the multiplicative update algorithms family, and it is necessary to include this feature to prove variation bounds on the regret.

\subsection{Discussion of the results}

Cesa-Bianchi et al. (2007) discussed desiderata for fundamental regret bounds for the expert prediction problem: invariance under translation and rescaling of costs vectors. Invariance under translation implies that the bounds depend only on the effective ranges of the cost vectors in each round, rather than the absolute ranges (by effective range, we mean the maximum difference between the costs in any given round). This is because of the following reason. If, in any given round, the costs of all experts are changed by the same amount, the difference between the expected cost of the algorithm in that round and the cost of any given expert remains the same as before. Our regret bounds enjoy this translation invariance property: this is a direct consequence of the variation bound. This implies, for instance, that it doesn't matter what sign the costs are, and in fact our bounds are robust enough to handle mixed signs in costs.

Rescaling invariance implies that the bound continues to hold even if all the cost vectors are scaled by the same factor. Again, our regret bounds enjoy rescaling invariance since the regret and the square-root variation scale by the same factors.

We make crucial use of these invariance properties in our analysis; the invariance allows us to normalize the cost vectors in ways that make them easier to reason about.

\subsection{Stationary stochastic vs. adversarial settings}

A point made by Cesa-Bianchi et al. (2007) is that the variation bounds on the regret essentially match the performance of a natural algorithm in the stochastic setting in which the 
payoffs are generated by a stationary stochastic process. Let us give a rough sketch of why this is true. Consider a setting of online linear optimization over the unit ball. Suppose that the cost functions are generated by a stationary stochastic process, such that in each iteration the cost function is independently sampled from a fixed distribution with some mean vector $\mu$. For a long enough sequence of cost functions drawn from this distribution, the best point in hindsight is essentially the least cost point with respect to the cost vector $\mu$.

Let $\bar{\mu}$ be the observed mean of samples. The natural algorithm uses $\bar{\mu}$ as proxy for the actual mean and chooses its point with $\bar{\mu}$ as a cost vector, and this can be shown to be optimal. It is a standard fact that the variance of $\bar{\mu}$ decreases inversely with the number of samples. Thus, if $\sigma^{2}$ is the variance of the distribution, then the variance of $\bar{\mu}$ after $t$ iterations is $\frac{\sigma^{2}}{t}$. The expected regret on iteration $t$ is proportional to the standard deviation $\frac{\sigma}{\sqrt{t}}$, and thus the total regret of the optimal predictor is on the order of $\sum_{t=1}^{T} \frac{\sigma}{\sqrt{t}}=O\left(\sqrt{\sigma^{2} T}\right)=O\left(\sqrt{\mathrm{VAR}_{T}}\right)$.

Hence, the optimal achievable regret in this simple setting is proportional to square root of the total variation. In the sequel we prove that the same regret (up to constant factors) can be achieved in the fully adversarial setting, i.e. in a setting in which the cost functions are chosen completely adversarially. In the stationary stochastic setting, the average cost converges to the average optimum cost at a speed that depends on the variance of the distribution: lower variance implies faster convergence. Hence, by proving the variation bounds on the regret, we give strong indication that online linear optimization in the adversarial setting is as efficient as in the stationary stochastic setting.

\subsection{A brief history of prediction}

It is incredible that as early as the late fifties, Hannan (1957) devised an efficient algorithm for prediction with expert advice. Hannan's algorithm proceeds by adding a perturbation to the costs of experts seen so far, and choosing the expert with least cost (taking into account the perturbations). He proves that the regret of an online player using his algorithm grows like $O(\sqrt{T})$ where $T$ is the number of prediction iterations.

Since then, there has been much progress on the expert prediction problem, and its extensions to online decision making: this includes the aforementioned influential multiplicative update family of algorithms (Littlestone and Warmuth 1994; Vovk 1998; Freund and Schapire 1997), Cover's universal portfolio prediction problem (Cover 1991) and the extensions of Follow-The-Perturbed-Leader (Kalai and Vempala 2005) to online optimization and complex decision problems such as online shortest paths. The machine learning community has extended these fundamental results into a beautiful theory of general prediction using Bregman divergences and generalized projections (in order to do justice to the numerous contributors we refer the reader to the comprehensive book Cesa-Bianchi and Lugosi 2006). This work refined upon the basic regret bound of $O(\sqrt{T})$. This refinement, however, deals with the constants multiplying the $\sqrt{T}$ term.

Freund and Schapire (1997) showed that a Multiplicative Weights algorithm based on the Weighted Majority algorithm attains regret bounds of $O\left(\sqrt{R \sum_{t=1}^{T} f_{t}\left(i^{*}\right) \log n}\right)$, where it is assumed that all costs are in the range $[0, R]$, and $i^{*}$ is the best expert in hindsight. In the case when the costs lie in the range $[-R, R]$, Allenberg-Neeman and Neeman (2004) showed that there is an expert $i$ such that the regret can be bounded by $O\left(\sqrt{R \sum_{t=1}^{T}\left|f_{t}(i)\right| \log n}\right)$. Most recently Cesa-Bianchi et al. (2007) gave the first second-order regret bounds: they proved a bound of $O\left(\sqrt{A_{T}^{\max } \log n}\right)$ where $A_{T}^{\max }=\max _{t \leq T}\left\{\sum_{\tau=1}^{t} f_{\tau}\left(\ell_{t}\right)^{2}\right\}$ is the maximum, over all the time periods $t$, of the sum of squares of losses up to time $t$ of the best expert at time $t$. 
They suggest, and indeed as we argue in the previous section it makes intuitive sense, that it should be possible to get a bound that scales as $\sqrt{\mathrm{VAR}_{T}^{\max }}$.

In this paper we prove their conjecture to be correct, in effect providing the optimal regret bounds up to constant factors.

\section{Notation and background}

The following definitions and derivations may be familiar to experts in learning theory, who may wish to proceed directly to the next section.

In the online linear optimization problem, the decision space is a closed, bounded, convex set $K \in \mathbb{R}^{n}$, and we are sequentially given a series of linear cost functions $f_{t}: K \rightarrow \mathbb{R}$ for $t=1,2, \ldots$ With some abuse of notation, we also write the functions as $f_{t}(x)=f_{t} \cdot x$ for some vector $f_{t} \in \mathbb{R}^{n}$.

The algorithm iteratively produces a point $x_{t} \in K$ in every round $t$, without knowledge of $f_{t}$ (but using the past sequence of cost functions), and incurs the cost $f_{t}\left(x_{t}\right)$. The regret at time $T$ is defined to be

$$
\operatorname{Regret}\left(f_{1}, f_{2}, \ldots, f_{T}\right):=\sum_{t=1}^{T} f_{t}\left(x_{t}\right)-\min _{x \in K} \sum_{t=1}^{T} f_{t}(x) .
$$

Usually, we will drop the cost vectors from the regret notation when they are clear from context. For convenience, we define $f_{0}=0$, and let $F_{t}=\sum_{\tau=0}^{t-1} f_{\tau}$.

We proceed to describe a widely used algorithmic technique in online learning, on the basis of which we will derive our algorithms.

Since our goal is to get regret bounded by the variation in the cost sequence, intuitively, a Follow-The-Leader (FTL) type algorithm, which always chooses the best point so far to use in the next round, should perform well if the variation is low. The FTL algorithm by itself doesn't usually guarantee low regret, mainly because it is inherently unstable: it may swing wildly from one point to another from one iteration to the next at very little provocation (for example, consider the case of expert prediction with 2 experts for the following sequence of cost vectors: $(1 / 2,0),(0,1),(1,0),(0,1), \ldots)$. To make it stable, we add a strictly convex regularization function $R(x)$ before computing the leader. The generic algorithm which results is shown below, and is called Follow The Regularized Leader (FTRL):

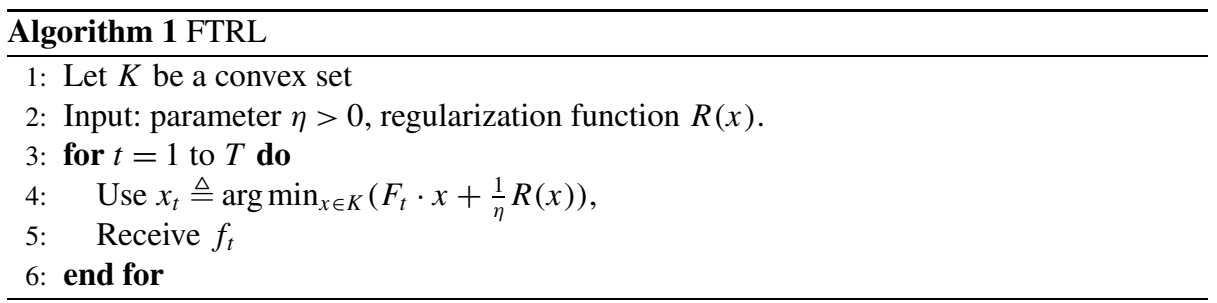

A crucial observation regarding the FTRL algorithm which we use in the analysis is its equivalence to the following algorithm, which we call Follow the Lazy Projected Leader (FLPL). This algorithm maintains an auxiliary sequence of points which are updated using 
a gradient descent type algorithm, which are then projected into the convex set using the Bregman divergence $B^{R}$ defined by $R$ :

$$
B^{R}(x, y)=R(x)-R(y)-\nabla R(y) \cdot(x-y) .
$$

The algorithm as it is given has an implicit update, whose implementation we ignore for now (in this paper we are only concerned with the Euclidean and Relative Entropy divergences, in which case the updates are efficient).

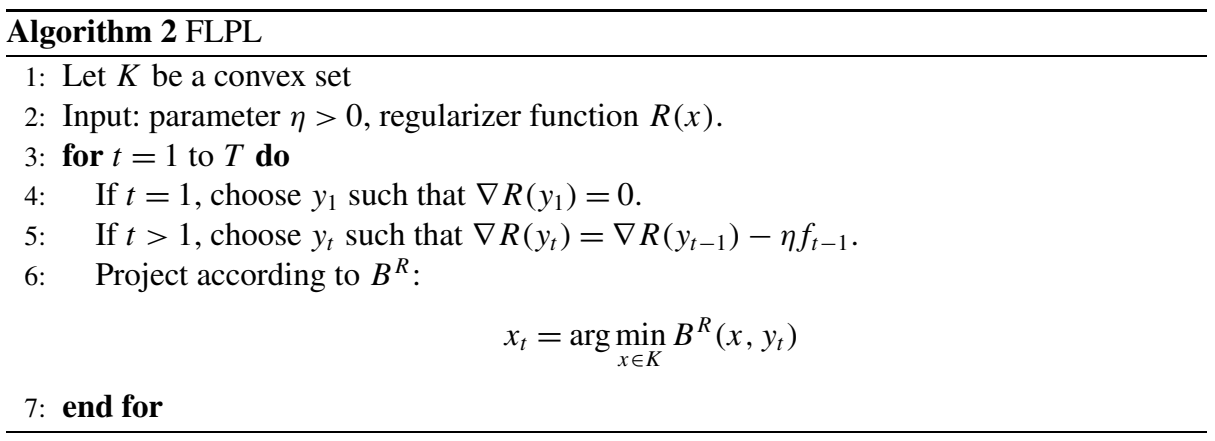

In fact, the two algorithms above are identical. This is perhaps not surprising, given what is known about the so called "mirror-descent" algorithm (e.g. Cesa-Bianchi and Lugosi 2006). Nevertheless this fact is crucial for our later derivations, and we did not find this precise statement elsewhere, hence we include a short proof.

Lemma 1 The two algorithms above produce identical predictions, i.e.

$$
\arg \min _{x \in K}\left(F_{t} \cdot x+\frac{1}{\eta} R(x)\right)=\arg \min _{x \in K} B^{R}\left(x, y_{t}\right)
$$

Proof First, let us observe that the unconstrained optimum $x^{*}=\arg \min _{x \in \mathbb{R}^{n}}\left(F_{t} \cdot x+\frac{1}{\eta} R(x)\right)$ satisfies

$$
F_{t}+\frac{1}{\eta} \nabla R\left(x^{*}\right)=0
$$

By induction, the above equation is also satisfied for $y_{t}$. Since $R(x)$ is assumed to be strictly convex, there is only one solution for the above equation and thus $y_{t}=x^{*}$. Hence,

$$
\begin{aligned}
B_{R}\left(x, y_{t}\right) & =R(x)-R\left(y_{t}\right)-\nabla R\left(y_{t}\right) \cdot\left(x-y_{t}\right) \\
& =R(x)-R\left(y_{t}\right)+\eta F_{t} \cdot\left(x-y_{t}\right) .
\end{aligned}
$$

Since $R\left(y_{t}\right)$ and $F_{t} \cdot y_{t}$ are constants (i.e. independent of $\left.x\right), B_{R}\left(x, y_{t}\right)$ is minimized at the point $x$ that minimizes $R(x)+\eta F_{t} \cdot x$, which implies that

$$
\arg \min _{x \in K} B_{R}\left(x, y_{t}\right)=\arg \min _{x \in K}\left(F_{t} \cdot x+\frac{1}{\eta} R(x)\right) .
$$


One important property which follows from the first characterization of $x_{t}$ is the following standard bound on the regret, due to Kalai and Vempala (2005), called the Follow-TheLeader/Be-The-Leader (FTL-BTL) inequality:

Lemma 2 The regret of the FTRL (or equivalently, the FLPL) algorithm is bounded as:

$$
\text { Regret } \leq \sum_{t=1}^{T} f_{t} \cdot\left(x_{t}-x_{t+1}\right)+\frac{1}{\eta}\left[\max _{x \in K} R(x)-R\left(x_{0}\right)\right] .
$$

Proof For convenience of notation, define the period 0 cost function $f_{0}: K \rightarrow \mathbb{R}$ as $f_{0}(x)=$ $\frac{1}{\eta} R(x)$. Also, with some abuse of notation, we use the notation $f_{t}(x)$ to also mean the linear functions defined by the vector $f_{t}$, viz. $f_{t}(x)=f_{t} \cdot x$. Then the FTRL algorithm can be succinctly described as choosing the points $x_{t}=\arg \min _{x \in K} \sum_{\tau=0}^{t-1} f_{t}(x)$.

We prove by induction that for any $t \geq 0$, we have

$$
\sum_{\tau=0}^{t} f_{\tau}\left(x_{\tau+1}\right) \leq \min _{x \in K} \sum_{\tau=0}^{t} f_{t}(x)
$$

whence the regret bound follows because if $x^{*}=\arg \min _{x \in K} \sum_{\tau=1}^{T} f_{\tau}(x)$, we have

$$
\text { Regret }=\sum_{\tau=1}^{T} f_{\tau}\left(x_{\tau}\right)-f_{\tau}\left(x^{*}\right) \leq \sum_{\tau=1}^{T}\left[f_{\tau}\left(x_{\tau}\right)-f_{\tau}\left(x_{\tau+1}\right)\right]+f_{0}\left(x^{*}\right)-f_{0}\left(x_{0}\right) .
$$

The statement for $t=0$ is obvious since $x_{1}=\arg \min _{x \in K} f_{0}(x)$. So assume the statement is true for $t \geq 0$, and we now proceed to prove it for $t+1$. We have

$$
\sum_{\tau=0}^{t+1} f_{\tau}\left(x_{\tau+1}\right) \leq \min _{x \in K} \sum_{\tau=0}^{t} f_{t}(x)+f_{t}\left(x_{t+1}\right),
$$

by induction hypothesis. Furthermore, we have

$$
\min _{x \in K} \sum_{\tau=0}^{t} f_{t}(x)+f_{t}\left(x_{t+1}\right) \leq \sum_{\tau=0}^{t} f_{t}\left(x_{t+1}\right)+f_{t}\left(x_{t+1}\right)=\min _{x \in K} \sum_{\tau=0}^{t} f_{t}(x) .
$$

Thus, the induction is complete.

\section{Algorithms and main results}

In this section we describe the algorithms for which we prove variation bounds, and state formally their performance guarantees.

\subsection{Online linear optimization}

We start by describing our result for online linear optimization. Following the notation defined in the previous section, we assume that $K \subseteq \mathbb{B}_{n}$, where $\mathbb{B}_{n}$ is the unit ball in $\mathbb{R}^{n}$, and that $0 \in K$. This is without loss of generality, and can be assumed by a suitable scaling and translation of $K$. Scaling $K$ down by its diameter $D$ makes the diameter 1 and scales the 
regret down by $D$ as well, and changing the coordinate system so that $K$ contains the origin doesn't change the regret bound. Here, we are making use of the translation invariance of our regret bounds.

We also assume that for all $t,\left\|f_{t}\right\| \leq 1$. If we have some other bound $R$ on $\left\|f_{t}\right\|$, then we scale down the $f_{t}$ 's by $R$ to get new cost vectors $f_{t}^{\prime}$ such that $\left\|f_{t}^{\prime}\right\| \leq 1$. We can then run the algorithm pretending as if $f_{t}^{\prime}$ is the sequence of cost vectors.

Define the variation of sequence of cost vectors $f_{1}, \ldots, f_{T}$ to be

$$
\operatorname{VAR}_{T}\left(f_{1}, f_{2}, \ldots, f_{T}\right)=\sum_{t=1}^{T}\left\|f_{t}-\mu\right\|^{2},
$$

where $\mu=\frac{1}{T} \sum_{t=1}^{T} f_{t}$ is the vector that minimizes the above expression. Usually, we will drop the cost vectors from the notation for the variation, and refer to it simply as $\mathrm{VAR}_{T}$, when the cost vectors are clear from context. To see that scaling has no effect on the regret bound, note that

$$
\operatorname{VAR}_{T}\left(f_{1}^{\prime}, \ldots, f_{T}^{\prime}\right)=\frac{1}{R^{2}} \operatorname{VAR}_{T}\left(f_{1}, \ldots, f_{T}\right),
$$

and

$$
\operatorname{Regret}\left(f_{1}^{\prime}, \ldots, f_{T}^{\prime}\right)=\frac{1}{R} \operatorname{Regret}\left(f_{1}, \ldots, f_{T}\right) .
$$

Thus, if $\operatorname{Regret}\left(f_{1}^{\prime}, \ldots, f_{T}^{\prime}\right)=O\left(\sqrt{\operatorname{VAR}_{T}\left(f_{1}^{\prime}, \ldots, f_{T}^{\prime}\right)}\right)$, then $\operatorname{Regret}\left(f_{1}, \ldots, f_{T}\right)=$ $O\left(\sqrt{\operatorname{VAR}_{T}\left(f_{1}, \ldots, f_{T}\right)}\right)$. This is exactly the rescaling invariance discussed earlier.

For ease of notation, we define $f_{0}=0$, and for any $t>0$, let $F_{t}=\sum_{\tau=0}^{t-1} f_{\tau}$ and $\mu_{t}=$ $\frac{1}{t} F_{t}=\frac{1}{t} \sum_{\tau=0}^{t-1} f_{\tau}$. We instantiate the FTRL/FLPL algorithm with the regularization function $R(x)=\frac{1}{2}\|x\|^{2}$. This regularization was considered many times before, and the only change here is to choose a different "learning rate" $\eta$, which will enable us to prove the novel regret bounds. Since $\nabla R(x)=x$ for this regularization, the algorithm that results is:

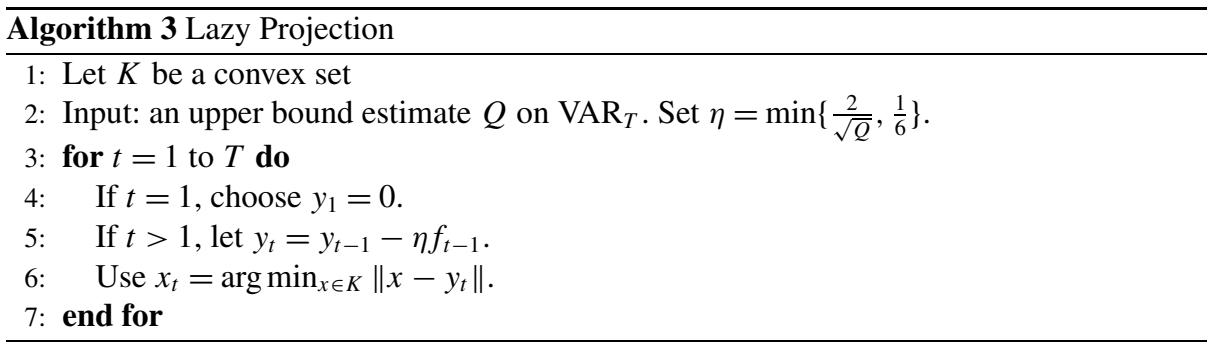

Our main theorem with respect to online linear optimization is:

Theorem 3 Let $f_{t}$, for $t=1,2, \ldots, T$, be a sequence of cost vectors such that $\left\|f_{t}\right\| \leq 1$. If the upper bound estimate $Q$ on $V A R_{T}$ is accurate, then the regret of the Lazy Projection algorithm is bounded by

$$
\text { Regret } \leq \min \{15 \sqrt{Q}, 150\}
$$

The constants in the bound are not optimized. Similar bounds for the case in which the total variation $\operatorname{VAR}_{T}$ is not known in advance are given in Sect. 3.3. 


\subsection{Prediction from expert advice}

In the expert learning problem, we assume that we have access to $n$ experts. In each round $t$, we choose a distribution $x_{t}$ over the experts and choose an expert from it. Then, we obtain a cost vector $f_{t}$ which specifies a cost $f_{t}(i)$ for every expert, and we incur the cost of the chosen expert. Our goal is to bound the total expected cost of the algorithm (i.e. $\sum_{t=1}^{T} f_{t} \cdot x_{t}$ ) relative to the total cost of the expert with minimum total cost in hindsight (i.e. $\left.\min _{i} \sum_{t=1}^{T} f_{t}(i)\right)$.

For simplicity, we assume that all costs $f_{t}(i) \in[0,1]$. This can be assumed without loss of generality from the rescaling and translation invariance of our final regret bounds. In general, all we need is a bound $R$ on the maximum value of $f_{t}(i)-f_{t}(j)$ over all rounds and all pairs of experts $i, j$. In each round, the algorithm can be run by scaling the costs of all experts down by $R$, and then subtracting out the minimum cost in each round. As in the case of online linear optimization, this scaling and translation doesn't affect the square-root variation bound on the regret.

As mentioned before, this setting is a special case of the online linear optimization where the domain $K$ is the simplex (denoted $\Delta$ ) of distributions over the experts. To design an algorithm for this special case, we need a different regularization function, ne $(x)=$ $\sum_{i} x_{i} \ln x_{i}-x_{i}$. The Bregman divergence which arises from this is the un-normalized relative entropy (c.f. Herbster and Warmuth 2001), defined on $\mathbb{R}_{+}^{n}$, called as follows:

$$
D_{\mathrm{ne}}(x, y):=\sum_{i} y_{i} \cdot \ln \frac{y_{i}}{x_{i}}+y_{i}-x_{i}
$$

Note that when $x, y \in \Delta, D_{\text {ne }}(x, y)$ is the relative entropy between $x$ and $y$, and ne $(x)$ is the negative entropy of $x$. The Bregman projection on the simplex with the un-normalized relative entropy divergence is implemented simply by scaling all the coordinates so that they sum to 1 .

A significant twist on the usual multiplicative weights algorithm is that we modify the cost functions to explicitly take into account the variation: we actually run the FTRL/FLPL algorithm on the sequence of cost vectors $\tilde{f}_{1}, \tilde{f}_{2}, \ldots$ where

$$
\tilde{f}_{t}(i)=\left[f_{t}(i)+4 \eta\left(f_{t}(i)-\mu_{t}(i)\right)^{2}\right]
$$

where $\mu_{t}=\frac{1}{t} \sum_{\tau=0}^{t-1} f_{\tau}$. As before, we use the convention that $f_{0}=0$.

For ease of notation, for a vector $x$, we define the vector $x^{2}$ as $x^{2}(i)=x(i)^{2}$. Thus, we can write $\tilde{f}_{t}$ compactly as $\tilde{f}_{t}=f_{t}+4 \eta\left(f_{t}-\mu_{t}\right)^{2}$. The algorithm which results is given below:

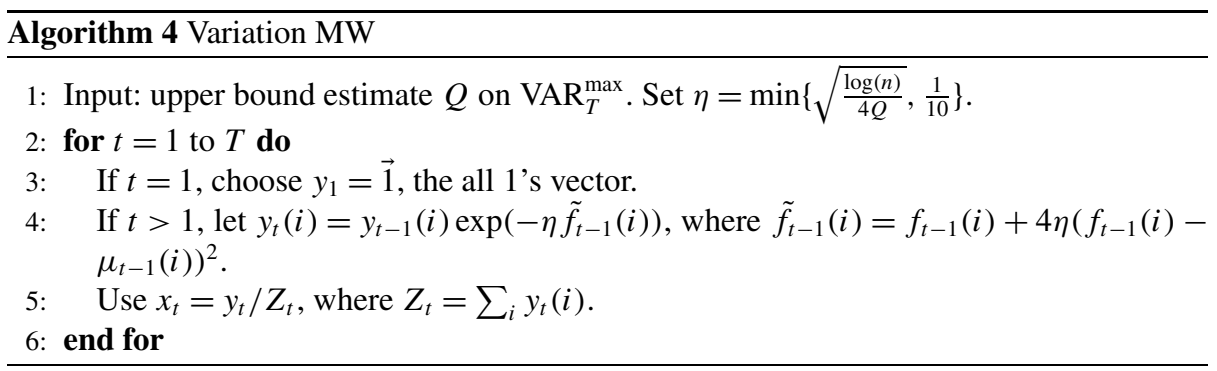


Define

$$
\operatorname{VAR}_{T}^{\max }=\max _{t \leq T}\left\{\operatorname{VAR}_{t}\left(\ell_{t}\right)\right\},
$$

where $\ell_{t}$ is the best expert till the $t^{\text {th }}$ round, and $\operatorname{VAR}_{t}(i)=\sum_{\tau=1}^{t}\left(f_{t}(i)-\mu_{t}^{\star}(i)\right)^{2}$ where $\mu_{t}^{\star}(i)=\frac{1}{t} \sum_{\tau=1}^{t} f_{\tau}(i)$ is the mean cost of the $i^{\text {th }}$ expert till the $t^{\text {th }}$ round. Our main result concerning prediction from expert advice is the following:

Theorem 4 Let $f_{t}$, for $t=1,2, \ldots, T$, be a sequence of cost vectors to the experts so that $f_{t}(i) \in[0,1]$. If the upper bound estimate $Q$ on $\mathrm{VAR}_{T}^{\max }$ is accurate, then the regret of the Variation $M W$ algorithm is bounded by

$$
\text { Regret } \leq 8 \sqrt{Q \log (n)}+10 \log (n) .
$$

Note that the additive $\log (n)$ term is inherent to expert learning algorithms and also appears in all previously known regret bounds. Again, one can obtain the same bounds up to constants even in the case in which $\mathrm{VAR}_{T}^{\max }$ is not known ahead of time. This is detailed in the next subsection.

\subsection{Bounds which hold uniformly over time}

Both of our main theorems for the settings of online linear optimization and prediction from expert advice have analogues to the case in which the variation is not known a priori. The generalization is a straightforward application of a technique called "the doubling trick", which we include here for completeness. It was applied previously, for example, in Auer et al. (2003).

First we introduce this technique in a very general form, so as to derive generalizations to both our previous theorems in a unified manner. Let $V_{T}=V\left(f_{1}, \ldots, f_{T}\right)$ be a monotone non-decreasing function of a sequence of cost functions (for example, the number of prediction periods $T$, or the two notions of variation from previous sections). We assume without loss of generality that $V_{T}$ can grow by at most 1 in every iteration. Consider an online prediction setting in which the predictor has access to an online algorithm called ALG, which, when supplied with an upper bound estimate $Q$ on $V$ guarantees the following regret bound:

$$
\text { Regret } \leq c_{1} \sqrt{Q}+c_{2} .
$$

Here, $c_{1}$ and $c_{2}$ are constants.

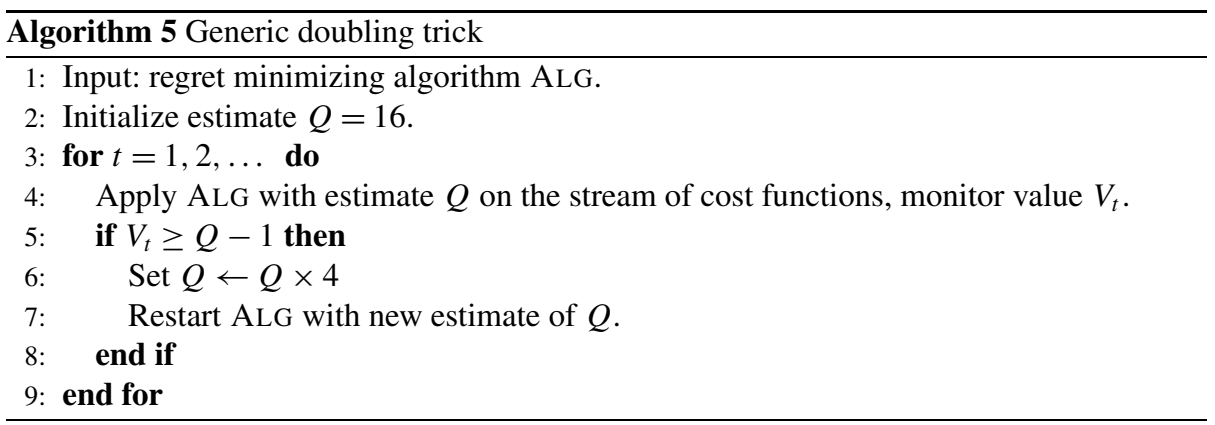


Lemma 5 Assume $V_{T} \geq 4$. Applying Algorithm 5, the regret of the resulting algorithm for any sequence of cost functions whose magnitude is bounded by one is bounded by

$$
\text { Regret } \leq 8 c_{1} \sqrt{V_{T}}+2 c_{2} \log \left(V_{T}\right) .
$$

Proof Imagine Algorithm 5 as running in phases $i=1,2, \ldots$, where in phase $i$, ALG is applied with the estimate $4^{i+1}$. Phase $i$ ends as soon as $V_{t}$ becomes at least $4^{i+1}-1$. Clearly, there are at most $\left\lfloor\log _{4}\left(V_{T}\right)\right\rfloor+1$ phases in all. At the end of the $i^{\text {th }}$ phase the function $V_{t}$ can be bounded by $V_{t-1}+1<4^{i+1}$, and thus by the assumption on ALG, the regret of the online algorithm is bounded by $c_{1} \sqrt{4^{i+1}}+c_{2}=c_{1} \cdot 2^{i+1}+c_{2}$. Thus, the total regret is bounded by:

$$
\sum_{i=1}^{\left\lfloor\log _{4} Q\right\rfloor+1} c_{1} \cdot 2^{i+1}+c_{2} \leq 8 c_{1} \sqrt{V_{T}}+2 c_{2} \log _{4}\left(V_{T}\right) .
$$

Here, we use the fact that since $V_{T} \geq 4$, we have $\left\lfloor\log _{4}\left(V_{T}\right)\right\rfloor+1 \leq 2 \log _{4}\left(V_{T}\right)$.

As a corollary of this lemma and our main Theorems 3 and 4 we have

Theorem 6 Let $f_{t}$, for $t=1,2, \ldots$, be a sequence of cost vectors to the experts so that $\left\|f_{t}\right\| \leq 1$. There exists an algorithm such that for all time steps $T$ the regret is bounded by

$$
\text { Regret } \leq \min \left\{120 \sqrt{\operatorname{VAR}_{T}}, 450\right\} .
$$

Proof This theorem doesn't follow directly from Lemma 5 because of the min function in the regret bound of Theorem 3. However, we can still apply the same generic algorithm 5, and after the third phase, the variation is large enough that the regret can be uniformly bounded (via Theorem 3) as

$$
\text { Regret } \leq 15 \sqrt{Q},
$$

at which point we can apply Lemma 5. The constant regret of 150 in the first 3 phases accounts for the 450 term in the bound.

Theorem 7 Let $f_{t}$, for $t=1,2, \ldots, T$, be a sequence of cost vectors to the experts so that $f_{t}(i) \in[0,1]$. There exists an algorithm such that for all time steps $T$ the regret is bounded by

$$
\text { Regret } \leq 64 \sqrt{\operatorname{VAR}_{T}^{\max } \log (n)}+20 \log (n) \log \mathrm{VAR}_{T}^{\max } .
$$

Proof The theorem follows by direct application of Lemma 5.

\section{Analysis of the lazy projection algorithm}

In this section we prove Theorem 3. The proof uses the dual characterization of the FTRL type algorithms introduced previously: on one hand we follow the standard methodology of the Follow-The-Leader type algorithms, bounding the regret by distance between consecutive predictions. On the other hand we use the fact that these predictions are projections of aggregate cost functions, and analyze the distance between successive projections. In fact, 
this latter analysis is the main crux of the proof-we refine previous approaches by giving a tighter bound on this distance which is based on simple geometrical intuition. Recall Theorem 3:

Theorem 3 Let $f_{t}$, for $t=1,2, \ldots, T$, be a sequence of cost vectors to the experts so that $\left\|f_{t}\right\| \leq 1$. If the upper bound estimate $Q$ on $V A R_{T}$ is accurate, then the regret of the Lazy Projection algorithm is bounded by

$$
\text { Regret } \leq \min \{15 \sqrt{Q}, 150\} .
$$

Proof In order to aid understanding, we present the proof as a series of lemmas. We defer the proofs of the lemmas to after the present proof. We start by invoking the FTL-BTL inequality (Lemma 2) to obtain the following bound:

\section{Lemma 8}

$$
\text { Regret } \leq \sum_{t=1}^{T}\left(f_{t}-\mu_{t}\right) \cdot\left(x_{t}-x_{t+1}\right)+\frac{1}{\eta} \text {. }
$$

We proceed to relate the distance between successive projections to the variation in the cost vectors. This lemma is the main crux of the proof, and is based on the geometric intuition depicted in Fig. 1. The idea in the proof is that if the sequence of cost vectors has low variation, then the cumulative cost vector $F_{t}$ is far away from the convex body, and in such a case, the distance between successive projections can be bounded in terms of the length of

Fig. 1 The distance between successive projections, viz. $\left\|x_{t}-x_{t+1}\right\|$, is bounded by the length of the component of $-\eta f_{t}$ orthogonal to the $y_{t}-x_{t}$

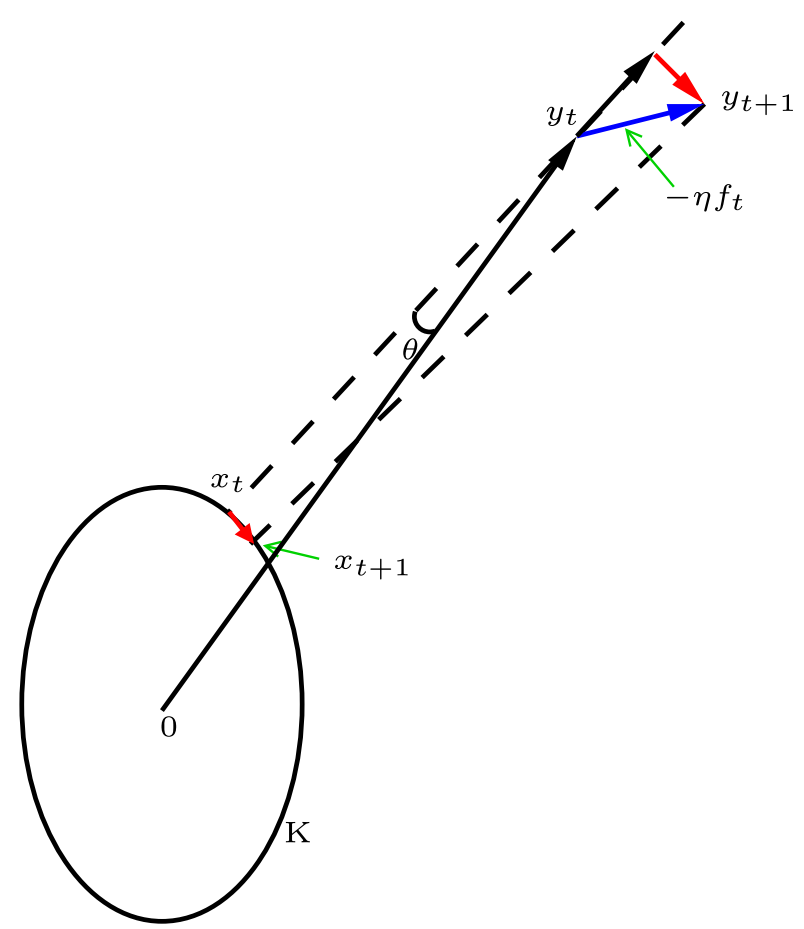


the component of $f_{t}$ orthogonal to $F_{t}$, which can in turn be bounded in terms of $\left\|f_{t}-\mu_{t}\right\|$, since $\mu_{t}=\frac{1}{t} F_{t}$.

Lemma 9 For all $t$, we have:

$$
\left\|x_{t}-x_{t+1}\right\| \leq \frac{3 \eta}{2}\left\|f_{t}-\mu_{t}\right\|+\frac{2}{t} .
$$

For ease of notation, we define a parameter of the cost vectors which will be further used in the analysis:

$$
\rho(T):=\sum_{t=1}^{T} \frac{1}{t}\left\|f_{t}-\mu_{t}\right\| .
$$

This parameter measures the variation of the cost vectors. Using the Cauchy-Schwartz inequality and Lemma 9 we get

$$
\begin{aligned}
& \left(f_{t}-\mu_{t}\right) \cdot\left(x_{t}-x_{t+1}\right) \\
& \quad \leq\left\|f_{t}-\mu_{t}\right\| \cdot\left[\frac{3 \eta}{2}\left\|f_{t}-\mu_{t}\right\|+\frac{2}{t}\right] \\
& \quad \leq \frac{3 \eta}{2}\left\|f_{t}-\mu_{t}\right\|^{2}+\frac{2\left\|f_{t}-\mu_{t}\right\|}{t} .
\end{aligned}
$$

Plugging this into the regret bound of Lemma 8 gives us the following bound:

$$
\text { Regret } \leq \frac{3 \eta}{2} \sum_{t=1}^{T}\left\|f_{t}-\mu_{t}\right\|^{2}+2 \rho(T)+\frac{1}{\eta} .
$$

To proceed from here, we use the following Lemma (which, curiously enough, is proved using the analysis of an online learning algorithm that has nothing to do with the present setting!):

Lemma 10 For any vector $\mu$, we have:

$$
\sum_{t=1}^{T}\left\|f_{t}-\mu_{t}\right\|^{2} \leq \sum_{t=1}^{T}\left\|f_{t}-\mu\right\|^{2}+4 \rho(T) .
$$

Plugging into (1) we get that, for any vector $\mu$ (and in particular, for $\mu=\mu_{T}^{\star}:=\frac{1}{T} \sum_{t=1}^{T} f_{t}$ ),

$$
\text { Regret } \leq \frac{3 \eta}{2} \sum_{t=1}^{T}\left\|f_{t}-\mu\right\|^{2}+(2+6 \eta) \rho(T)+\frac{1}{\eta} .
$$

We can bound $\rho(T)$ as follows:

Lemma 11 For any vector $\mu$, we have:

$$
\rho(T) \leq 3 \sqrt{\sum_{t=1}^{T}\left\|f_{t}-\mu\right\|^{2}} .
$$


Thus, if the upper bound $Q$ on $\operatorname{VAR}_{T}$ holds, we have

$$
\text { Regret } \leq \frac{3 \eta}{2} Q+(6+18 \eta) \sqrt{Q}+\frac{1}{\eta} .
$$

Finally, by setting $\eta=\min \{2 / \sqrt{Q}, 1 / 6\}$, the proof is complete.

We now give the omitted proofs of lemmas used in the above proof.

Proof of Lemma 8 By definition of $x_{t}$, we know that

$$
F_{t} \cdot x_{t}+\frac{1}{2 \eta}\left\|x_{t}\right\|^{2} \leq F_{t} \cdot x_{t+1}+\frac{1}{2 \eta}\left\|x_{t+1}\right\|^{2} .
$$

Recall that $\mu_{t}=F_{t} / t$. Hence,

$$
\begin{aligned}
\sum_{t=1}^{T} \mu_{t} \cdot\left(x_{t}-x_{t+1}\right) & =\sum_{t=1}^{T} \frac{F_{t}}{t} \cdot\left(x_{t}-x_{t+1}\right) \\
& \leq \sum_{t=1}^{T} \frac{1}{t} \cdot \frac{1}{2 \eta}\left(\left\|x_{t+1}\right\|^{2}-\left\|x_{t}\right\|^{2}\right) \\
& \leq \frac{1}{2 \eta} \sum_{t=2}^{T}\left\|x_{t}\right\|^{2} \cdot\left(\frac{1}{t-1}-\frac{1}{t}\right)+\frac{\left\|x_{T+1}\right\|^{2}}{2 \eta T} \\
& \leq \frac{1}{2 \eta} .
\end{aligned}
$$

Here, we use the fact that $\left\|x_{t}\right\| \leq 1$. The stated bound then follows from Lemma 2 .

Proof of Lemma 9 We split up the analysis in two cases:

1. $\left\|F_{t}\right\| \leq 2 / \eta$ : Assume that $\left\|F_{t}\right\|>0$. Since $x_{t}$ and $x_{t+1}$ are the projections of $y_{t}$ and $y_{t+1}$ respectively on $K$, by the Projection Lemma 12 we have

$$
\begin{aligned}
\left\|x_{t}-x_{t+1}\right\| & \leq\left\|y_{t}-y_{t+1}\right\| \\
& =\eta\left\|f_{t}\right\| \\
& \leq \eta\left\|f_{t}-\mu_{t}\right\|+\eta\left\|\mu_{t}\right\| \\
& \leq \eta\left\|f_{t}-\mu_{t}\right\|+\frac{2\left\|\mu_{t}\right\|}{\left\|F_{t}\right\|} \\
& =\eta\left\|f_{t}-\mu_{t}\right\|+\frac{2}{t} .
\end{aligned}
$$

If $\left\|F_{t}\right\|=0$, then the Projection Lemma 12 implies that $\left\|x_{t}-x_{t+1}\right\| \leq \eta\left\|f_{t}\right\|=$ $\eta\left\|f_{t}-\mu_{t}\right\|$, so the stated bound still holds.

2. $\left\|F_{t}\right\| \geq 2 / \eta$ : we first show the following bound:

$$
\left\|x_{t}-x_{t+1}\right\| \leq \eta\left\|f_{t}-\mu_{t}\right\|+\left\|f_{t}\right\| /\left\|F_{t}\right\|
$$


Consider two unit vectors: $u$ in the direction $y_{t}-x_{t}$, and $v$ in the direction $y_{t}$. We claim that the sine of the angle $\theta$ between these vectors is at most $1 / \eta\left\|F_{t}\right\|$. To see this, consider the triangle formed by the points $0, x_{t}, y_{t}$. We are interested in the angle $\theta$ at vertex $y_{t}$ (see Fig. 1). Let $\vartheta$ be the angle at $x_{t}$. By the law of sines, we have

$$
\sin (\theta)=\frac{\left\|x_{t}\right\| \sin (\vartheta)}{\left\|y_{t}\right\|} \leq \frac{1}{\left\|y_{t}\right\|}=\frac{1}{\eta\left\|F_{t}\right\|}
$$

where the inequality follows because $\left\|x_{t}\right\| \leq 1$ and $\sin (\vartheta) \leq 1$.

Now, we consider the components of $f_{t}$ along $u$ and $v$ : define $f_{t}^{u}=\left(f_{t} \cdot u\right) u$ and $f_{t}^{v}=\left(f_{t} \cdot v\right) v$. Consider the point $y_{t}-\eta f_{t}^{u}$. Since it lies on the line joining $y_{t}$ to $x_{t}$, its projection on $K$ is also $x_{t}$. Here, we use the fact that $y_{t}-\eta f_{t}^{u}$ is outside $K$ : this is because

$$
\left\|y_{t}-\eta f_{t}^{u}\right\| \geq\left\|y_{t}\right\|-\eta\left\|f_{t}^{u}\right\| \geq \eta\left\|F_{t}\right\|-\eta \geq 1 .
$$

By the Projection Lemma 12, we have

$$
\left\|x_{t+1}-x_{t}\right\| \leq\left\|y_{t+1}-\left(y_{t}-\eta f_{t}^{u}\right)\right\|=\eta\left\|f_{t}-f_{t}^{u}\right\| .
$$

Let $x$ be the projection of $f_{t}^{v}$ on the subspace spanned by $u$ (i.e. $x=\left(f_{t}^{v} \cdot u\right) u$ ). Then, since $f_{t}^{u}$ is the projection of $f_{t}$ in the subspace spanned by $u$, it is the closest point to $f_{t}$ in the subspace, and since $x$ is also in the subspace, we have

$$
\begin{aligned}
\left\|f_{t}-f_{t}^{u}\right\| & \leq\left\|f_{t}-x\right\| \\
& \leq\left\|f_{t}-f_{t}^{v}\right\|+\left\|f_{t}^{v}-x\right\| \\
& =\left\|f_{t}-f_{t}^{v}\right\|+\left\|f_{t}^{v}\right\| \sin (\theta) \\
& \leq\left\|f_{t}-f_{t}^{v}\right\|+\left\|f_{t}\right\| / \eta\left\|F_{t}\right\| \\
& \leq\left\|f_{t}-\mu_{t}\right\|+\left\|f_{t}\right\| / \eta\left\|F_{t}\right\| .
\end{aligned}
$$

The last inequality follows because $f_{t}^{v}$ is the closest point to $f_{t}$ in the subspace spanned by $v$, and $\mu_{t}$ is a point in this subspace. Plugging this bound into (3), we get (2).

Now, we have the following bound on $\left\|f_{t}\right\| /\left\|F_{t}\right\|$ :

$$
\frac{\left\|f_{t}\right\|}{\left\|F_{t}\right\|} \leq \frac{\left\|f_{t}-\mu_{t}\right\|+\left\|\mu_{t}\right\|}{\left\|F_{t}\right\|} \leq \frac{\eta}{2}\left\|f_{t}-\mu_{t}\right\|+\frac{1}{t} .
$$

Plugging (4) into (2), we get the required bound.

Proof of Lemma 10 We may assume that $\|\mu\| \leq 1$, since the right hand side is minimized at $\mu=\frac{1}{T} \sum_{t=1}^{T} f_{t}$. The statement of the lemma is essentially bounding the regret of the FTL algorithm played on the sequence of cost functions $c_{t}(x)=\left\|x-f_{t}\right\|^{2}$, for $t=0,1,2, \ldots, T$, with the convex domain the unit ball $\mathbb{B}_{n}$. This is because the leader in round $t$ is

$$
\arg \min _{x \in \mathbb{B}_{n}}\left\{\sum_{\tau=0}^{t-1}\left\|x-f_{\tau}\right\|^{2}\right\}=\frac{1}{t} \sum_{\tau=0}^{t-1} f_{\tau}=\mu_{t} .
$$


We assume here that the first point played by the algorithm is 0 . Then by the FTL-BTL inequality (Lemma 2), the regret of the FTL algorithm can be bounded as (here, the regularization function $R(x)$ is null):

$$
\begin{aligned}
\text { Regret } & \leq c_{0}(0)-c_{0}\left(\mu_{1}\right)+\sum_{t=1}^{T} c_{t}\left(\mu_{t}\right)-c_{t}\left(\mu_{t+1}\right) \\
& \leq \sum_{t=1}^{T} \nabla c_{t}\left(\mu_{t}\right) \cdot\left(\mu_{t}-\mu_{t+1}\right) \quad\left(\because c_{t} \text { is convex }\right) \\
& \leq \sum_{t=1}^{T}\left\|\nabla c_{t}\left(\mu_{t}\right)\right\|\left\|\mu_{t}-\mu_{t+1}\right\| \\
& \leq \sum_{t=1}^{T}\left\|2\left(f_{t}-\mu_{t}\right)\right\| \cdot\left\|\mu_{t}-\mu_{t+1}\right\| .
\end{aligned}
$$

Now, we have

$$
\left\|\mu_{t}-\mu_{t+1}\right\|=\left\|\mu_{t}-\frac{t \mu_{t}+f_{t}}{t+1}\right\| \leq \frac{1}{t+1}\left(\left\|\mu_{t}\right\|+\left\|f_{t}\right\|\right) \leq \frac{2}{t} .
$$

Thus, the regret is bounded by $4 \rho(T)$.

Proof of Lemma 11 We may assume without loss of generality that $\mu=0$ : using the vectors $f_{t}-\mu$ instead of $f_{t}$ doesn't change the value of $\rho(T)$. We have

$$
\begin{aligned}
\rho(T) & =\sum_{t=1}^{T} \frac{1}{t}\left\|f_{t}-F_{t} / t\right\| \\
& \leq \sum_{t=1}^{T} \frac{1}{t}\left[\left\|f_{t}\right\|+\frac{1}{t}\left\|F_{t}\right\|\right] \\
& \leq \sum_{t=1}^{T}\left[\frac{1}{t}\left\|f_{t}\right\|+\frac{1}{t^{2}} \sum_{\tau=1}^{t-1}\left\|f_{\tau}\right\|\right] \\
& \leq \sum_{t=1}^{T} \frac{2}{t}\left\|f_{t}\right\| \quad\left(\because \sum_{\tau=t+1}^{T} \frac{1}{t^{2}} \leq \frac{1}{t}\right) \\
& \leq \sqrt{\left[\sum_{t=1}^{T}\left\|f_{t}\right\|^{2}\right]\left[\sum_{t=1}^{T} \frac{4}{t^{2}}\right]} \text { (Cauchy-Schwarz) } \\
& \leq 3 \sqrt{\sum_{t=1}^{T}\left\|f_{t}\right\|^{2},}
\end{aligned}
$$

as required. 
The projection lemma which follows is a well-known fact from convex optimization theory. We include the proof for completeness.

Lemma 12 (Projection lemma) Let $K$ be a convex set, and let $x$ and $y$ be any two points. Let $x^{\prime}$ and $y^{\prime}$ be their respective projections on $K$. Then

$$
\left\|x^{\prime}-y^{\prime}\right\| \leq\|x-y\| .
$$

Proof Assume that $x^{\prime} \neq y^{\prime}$, otherwise the inequality is trivial. By the properties of projections on convex sets, we have

$$
\left(x-x^{\prime}\right) \cdot\left(y^{\prime}-x^{\prime}\right) \leq 0 \text { and }\left(y-y^{\prime}\right) \cdot\left(x^{\prime}-y^{\prime}\right) \leq 0 .
$$

Consider the line $\ell$ passing through $x^{\prime}$ and $y^{\prime}$, and consider the projections $x^{\prime \prime}$ and $y^{\prime \prime}$ of $x$ and $y$ respectively on this line. The inequalities (5) imply that along $\ell$, the order of the points is $\left(x^{\prime \prime}, x^{\prime}, y^{\prime}, y^{\prime \prime}\right)$. Thus, we have

$$
\left\|x^{\prime}-y^{\prime}\right\| \leq\left\|x^{\prime \prime}-y^{\prime \prime}\right\| \leq\|x-y\|,
$$

where the last inequality follows because the projection of any line segment on any line is no longer than the segment itself.

\section{Analysis of the variation MW algorithm}

The analysis of the Variation MW is straightforward, though complicated somewhat due to heavy algebraic manipulations. We outline the main ideas in the analysis now. Our starting point is Lemma 13, a well-known bound which relates the regret of the Multiplicative Weights algorithm with the expected squared losses of the experts (the expectation being taken under the distributions generated by the algorithm). Next, we make crucial use of the fact that the Multiplicative Weighs algorithm puts exponentially higher weight on experts with lower cost than those with higher costs. Since we explicitly factor in the variation in the costs of each expert before computing their exponential weights, eventually the algorithm starts to concentrate all the weight on experts with lower cost and lower variation. This yields the desired regret bound.

We now describe a regret bound on the performance of the Multiplicative Weights algorithm. This bound is well-known (see, for e.g. Cesa-Bianchi et al. 2007; Auer et al. 2003), we include the short proof for completeness.

Lemma 13 Suppose in round $t$ of the expert prediction problem, expert $i$ incurs cost $g_{t}(i)$, where $\left|g_{t}(i)\right| \leq M$. Consider the Multiplicative Weights algorithm, that in round $t$ chooses expert $i$ with probability $x_{t}(i) \propto \exp \left(-\eta \sum_{\tau=1}^{t-1} g_{\tau}(i)\right)$. Then, if $\eta \leq 1 / M$,

$$
\text { Regret } \leq \eta \sum_{t=1}^{T} g_{t}^{2} \cdot x_{t}+\frac{\log n}{\eta} .
$$

Proof Let $w_{t}(i)=\exp \left(-\eta \sum_{\tau=1}^{t-1} g_{\tau}(i)\right)$, and let $Z_{t}=\sum_{i} w_{t}(i)$. Then the distribution on the experts at time $t$ is exactly $w_{t} / Z_{t}$. We think of $Z_{t}$ as a potential function, and track how it 
changes over time. Initially, $Z_{1}=n$. We have

$$
\begin{aligned}
Z_{t+1} & =\sum_{i} w_{t}(i) \exp \left(-\eta g_{t}(i)\right) \\
& \leq \sum_{i} w_{t}(i)\left(1-\eta g_{t}(i)+\eta^{2} g_{t}(i)^{2}\right) \\
& =Z_{t}\left(1-\eta\left(g_{t} \cdot x_{t}\right)+\eta^{2}\left(g_{t}^{2} \cdot x_{t}\right)\right) \\
& \leq Z_{t} \exp \left(-\eta\left(g_{t} \cdot x_{t}\right)+\eta^{2}\left(g_{t}^{2} \cdot x_{t}\right)\right) .
\end{aligned}
$$

In (6), we used the fact that for $|x| \leq 1$, we have $\exp (x) \leq 1+x+x^{2}$. Thus, by induction, we have

$$
Z_{T+1} \leq n \exp \left(-\eta \sum_{t=1}^{T}\left(g_{t} \cdot x_{t}\right)+\eta^{2} \sum_{t=1}^{T}\left(g_{t}^{2} \cdot x_{t}\right)\right) .
$$

Also, for any expert $i$ we have the bound

$$
Z_{T+1} \geq w_{T+1}(i)=\exp \left(-\eta \sum_{\tau=1}^{T} g_{\tau}(i)\right) .
$$

Putting these two inequalities together, taking logarithms and simplifying, we get the desired bound on the regret.

For our analysis, we use a slightly different notion of variation of the experts' costs: for any round $t$ and any expert $i$, define

$$
Q_{t}(i)=\sum_{\tau=1}^{t-1}\left(f_{\tau}(i)-\mu_{\tau}(i)\right)^{2} .
$$

Recall that the usual definition of variation of an experts cost up to the $t^{\text {th }}$ round is simply

$$
\operatorname{VAR}_{t}(i)=\sum_{\tau=1}^{t}\left(f_{\tau}(i)-\mu_{t}^{\star}(i)\right)^{2},
$$

where $\mu_{t}^{\star}(i)=\frac{1}{t} \sum_{\tau=1}^{t} f_{t}(i)$. But it is easily seen from (the 1 dimensional version of) Lemmas 10 and 11 that

$$
Q_{t}(i) \leq \operatorname{VAR}_{t}(i)+12 \sqrt{\operatorname{VAR}_{t}(i)}
$$

and thus $Q_{t}(i)$ can serve as a proxy for the true variation (up to constant factors).

Recall that $\ell_{t}$ is the best expert till time $t$, and $\operatorname{VAR}_{T}^{\max }=\max _{t \leq T}\left\{\operatorname{VAR}_{t}\left(\ell_{t}\right)\right\}$. Define $Q_{T}^{\max }=\max _{t \leq T} Q_{t}\left(\ell_{t}\right)$. Then, we have that

$$
Q_{T}^{\max } \leq 4 \mathrm{VAR}_{T}^{\max }
$$

assuming that $\mathrm{VAR}_{T}^{\max } \geq 16$. Then, the following Lemma combined with inequality (7) implies Theorem 4. 
Lemma 14 Let $f_{t}$, for $t=1,2, \ldots, T$, be a sequence of cost vectors to the experts so that $f_{t}(i) \in[0,1]$. Let $\ell_{t}$ be the best expert at time $t$, and let $Q$ be an upper bound on $Q_{T}^{\max }=$ $\max _{t}\left\{Q_{t}\left(\ell_{t}\right)\right\}$. Then setting $\eta=\min \{\sqrt{\log (n) / 4 Q}, 1 / 10\}$, the regret of the Variation $M W$ algorithm is bounded by

$$
\text { Regret } \leq 4 \sqrt{Q \log (n)}+10 \log (n) .
$$

Proof Define $g_{t}=\tilde{f}_{t}-\alpha_{t} \overrightarrow{1}$, where $\alpha_{t}=\mu_{t}\left(\ell_{t}\right)+\frac{4 \eta}{t} Q_{t}\left(\ell_{t}\right)$, and $\overrightarrow{1}$ is the all 1 's vector. Note that for any $i$,

$$
\exp \left(-\eta \sum_{\tau=1}^{t-1} g_{\tau}(i)\right)=\frac{1}{Z} \exp \left(-\eta \sum_{\tau=1}^{t-1} \tilde{f}_{\tau}(i)\right),
$$

where $Z$ is a scaling constant independent of $i$. Hence, scaling either the weights $\exp \left(-\eta \sum_{\tau=1}^{t-1} g_{\tau}(i)\right)$ or the weights $\exp \left(-\eta \sum_{\tau=1}^{t-1} \tilde{f}_{\tau}(i)\right)$ to sum up to 1 yields the same distribution, viz. $x_{t}$.

Since we assumed that the $f_{t}(i) \in[0,1]$, we conclude that $g_{t}(i) \in[-2,2]$ (since $\left.4 \eta \leq 1\right)$. Applying Lemma 13 to the sequence of cost vectors $g_{t}$, we get the following regret bound, where $\ell_{T}$ is the final best expert:

$$
\sum_{t=1}^{T} \tilde{f}_{t} \cdot x_{t}-\sum_{t=1}^{T} \tilde{f}_{t}\left(\ell_{T}\right) \leq \eta \sum_{t=1}^{T} g_{t}^{2} \cdot x_{t}+\frac{\log n}{\eta} .
$$

Here, we used the fact that the $\sum_{t=1}^{T} \alpha_{t} \overrightarrow{1} \cdot x_{t}=\sum_{t=1}^{T} \alpha_{t}$. Simplifying using the definition of $\tilde{f}_{t}$, we get

$$
\begin{aligned}
\sum_{t=1}^{T} f_{t} \cdot x_{t}-\sum_{t=1}^{T} f_{t}\left(\ell_{T}\right) \leq & \eta \sum_{t=1}^{T} g_{t}^{2} \cdot x_{t} \\
& +\frac{\log n}{\eta}-4 \eta \sum_{t=1}^{T}\left(f_{t}-\mu_{t}\right)^{2} \cdot x_{t}+4 \eta \sum_{t=1}^{T}\left(f_{t}\left(\ell_{T}\right)-\mu_{t}\left(\ell_{T}\right)\right)^{2} \\
\leq & \eta \sum_{t=1}^{T}\left[g_{t}^{2}-4\left(f_{t}-\mu_{t}\right)^{2}\right] \cdot x_{t}+4 \eta(Q+1)+\frac{\log n}{\eta}
\end{aligned}
$$

since $\sum_{t=1}^{T}\left(f_{t}\left(\ell_{T}\right)-\mu_{t}\left(\ell_{T}\right)\right)^{2} \leq Q_{T}\left(\ell_{T}\right)+1 \leq Q+1$.

The following lemma bounds the first term in (8). The proof is a straightforward calculation, and so we defer its proof to after the present proof.

Lemma 15 If $\eta \leq 1 / 10$, then for any $i$, we have

$$
g_{t}^{2}(i)-4\left(f_{t}(i)-\mu_{t}(i)\right)^{2} \leq 2\left(\mu_{t}(i)-\alpha_{t}\right)^{2} .
$$

Plugging this bound into (8), we get that

$$
\text { Regret } \leq 2 \eta \sum_{t=1}^{T}\left(\mu_{t}-\alpha_{t} \overrightarrow{1}\right)^{2} \cdot x_{t}+\frac{\log n}{\eta}+4 \eta(Q+1) \text {. }
$$


We now proceed to bound $\sum_{t=1}^{T}\left(\mu_{t}-\alpha_{t} \overrightarrow{1}\right)^{2} \cdot x_{t}$. We bound each term in the summation separately. For any $t \leq \frac{\log n}{\eta}$, we simply bound $\left|\mu_{t}(i)-\alpha_{t}\right| \leq 2$ and hence we have $\left(\mu_{t}-\right.$ $\left.\alpha_{t} \overrightarrow{1}\right)^{2} \cdot x_{t} \leq 2$.

Now let $t>\frac{\log n}{\eta}$. For convenience of notation, we drop the subscript $t$ from $x_{t}(i)$ and refer to them as $x(i)$.

$$
\begin{aligned}
\left(\mu_{t}-\alpha_{t} \overrightarrow{1}\right)^{2} \cdot x & =\sum_{i: \mu_{t}(i) \leq \alpha_{t}}\left(\mu_{t}(i)-\alpha_{t}\right)^{2} x(i)+\sum_{i: \mu_{t}(i)>\alpha_{t}}\left(\mu_{t}(i)-\alpha_{t}\right)^{2} x(i) \\
& \leq \sum_{i: \mu_{t}(i) \leq \alpha_{t}}\left[\frac{4 \eta}{t} Q_{t}\left(\ell_{t}\right)\right]^{2} x(i)+\sum_{i: \mu_{t}(i)>\alpha_{t}}\left(\mu_{t}(i)-\alpha_{t}\right)^{2} x(i) \\
& \leq\left[\frac{4 \eta}{t} Q_{t}\left(\ell_{t}\right)\right]^{2}+\sum_{i: \mu_{t}(i)>\alpha_{t}}\left(\mu_{t}(i)-\alpha_{t}\right)^{2} x(i) .
\end{aligned}
$$

Here, (10) follows because when $\mu_{t}(i) \leq \alpha_{t}=\mu_{t}\left(\ell_{t}\right)+\frac{4 \eta}{t} Q_{t}\left(\ell_{t}\right)$, we have $\left|\mu_{t}-\alpha_{t}\right| \leq$ $\frac{4 \eta}{t} Q_{t}\left(\ell_{t}\right)$ since $\mu_{t}(i) \geq \mu_{t}\left(\ell_{t}\right)$.

We now bound each term of (11) separately. The proof of the following lemma is a straightforward calculation and we defer it to after the present proof.

Lemma 16 The first term of (11), summed over all $t$, can be bounded as:

$$
\sum_{t=1}^{T}\left[\frac{4 \eta}{t} Q_{t}\left(\ell_{t}\right)\right]^{2} \leq 32 \eta^{2} Q
$$

The hard part is to bound the second term of (11). We now proceed to do so. The intuition in the following analysis is that the Variation MW algorithm tends to concentrate exponentially high weight on the experts that have low cost.

Let $I$ be the index set of all $i$ such that $\mu_{t}(i)>\alpha_{t}$. Note that $\ell_{t} \notin I$. Now, we have $x(i) \propto \exp \left(-\eta t \mu_{t}(i)-4 \eta^{2} Q_{t}(i)\right)$, and thus $x\left(\ell_{t}\right) \propto \exp \left(-\eta t \alpha_{t}\right)$. Thus, $x(i)$ can be written as:

$$
\begin{aligned}
x(i) & =\frac{\exp \left(-\eta t \mu_{t}(i)-4 \eta^{2} Q_{t}(i)\right)}{\exp \left(-\eta t \alpha_{t}\right)+\sum_{j \neq \ell_{t}} \exp \left(-\eta t \mu_{t}(j)-4 \eta^{2} Q_{t}(j)\right)} \\
& =\frac{\lambda(i) \exp \left(-\eta t\left(\mu_{t}(i)-\alpha_{t}\right)\right)}{1+\sum_{j \neq \ell_{t}} \lambda(j) \exp \left(-\eta t\left(\mu_{t}(j)-\alpha_{t}\right)\right)},
\end{aligned}
$$

where $\lambda(i)=\exp \left(-4 \eta^{2} Q_{t}(i)\right)$. Note that all $\lambda(i) \in(0,1]$. Define, for all $i, d(i)=$ $\left(\mu_{t}(i)-\alpha_{t}\right)$. Note that for $i \in I, d(i) \in[0,1]$. Thus, we have

$$
\sum_{i \in I} d(i)^{2} x(i)=\sum_{i \in I} \frac{\lambda(i) d(i)^{2} \exp (-\eta t d(i))}{1+\sum_{j \neq \ell_{t}} \lambda(j) \exp (-\eta t d(j))} .
$$

To upper bound $\sum_{i \in I} d(i)^{2} x(i)$, we can neglect the factors in the denominator which depend on $i \notin I \cup\left\{\ell_{t}\right\}$; this only increases the value. Let $d^{I}$ and $\lambda^{I}$ be the vectors $d$ and $\lambda$ restricted 
to the index set $I$. Define the function $h:(0,1]^{|I|} \times[0,1]^{|I|} \rightarrow \mathbb{R}$ as

$$
h\left(\lambda^{I}, d^{I}\right)=\sum_{i \in I} \frac{\lambda(i) d(i)^{2} \exp (-\eta t d(i))}{1+\sum_{j \in I} \lambda(j) \exp (-\eta t d(j))} .
$$

The maximum value of this function on its domain gives an upper bound on the expression above.

Lemma 17 For $t>\frac{\log n}{\eta}$, and for any $\left(\lambda^{I}, d^{I}\right) \in(0,1]^{|I|} \times[0,1]^{|I|}$, we have

$$
h\left(\lambda^{I}, d^{I}\right) \leq \frac{2 \log ^{2} n}{\eta^{2} t^{2}} .
$$

Putting Lemmas 16 and 17 together, we have that

$$
\begin{aligned}
\sum_{t=1}^{T}\left(\mu_{t}-\alpha_{t} \overrightarrow{1}\right)^{2} \cdot x_{t} & \leq \sum_{t \leq \frac{\log n}{\eta}} 2+\sum_{t=1}^{T}\left[\frac{4 \eta}{t} Q_{t}\left(\ell_{t}\right)\right]^{2}+\sum_{t>\frac{\log n}{\eta}} \frac{2 \log ^{2} n}{\eta^{2} t^{2}} \\
& \leq 32 \eta^{2} Q+\frac{4 \log n}{\eta} .
\end{aligned}
$$

Plugging this bound into (9), we get

$$
\text { Regret } \leq \frac{\log n}{\eta}+64 \eta^{3} Q+8 \log (n)+4 \eta(Q+1)
$$

Now, if we set $\eta=\{\sqrt{\log n / 4 Q}, 1 / 10\}$, we get that the regret is bounded by

$$
\text { Regret } \leq 4 \sqrt{Q \cdot \log n}+10 \log (n) .
$$

We now give the omitted proofs of Lemmas 15, 16, and 17.

Proof of Lemma 15 We have:

$$
\begin{aligned}
g_{t}(i)^{2} & =\left(f_{t}(i)-\alpha_{t}+4 \eta\left(f_{t}(i)-\mu_{t}(i)\right)^{2}\right)^{2} \\
& =\left(f_{t}(i)-\alpha_{t}\right)^{2}+8 \eta\left(f_{t}(i)-\alpha_{t}\right)\left(f_{t}(i)-\mu_{t}(i)\right)^{2}+16 \eta^{2}\left(f_{t}(i)-\mu_{t}(i)\right)^{4} \\
& \leq\left(f_{t}(i)-\alpha_{t}\right)^{2}+\left(16 \eta+16 \eta^{2}\right)\left(f_{t}(i)-\mu_{t}(i)\right)^{2} \\
& \leq 2\left(\mu_{t}(i)-\alpha_{t}\right)^{2}+\left(2+16 \eta+16 \eta^{2}\right)\left(f_{t}(i)-\mu_{t}(i)\right)^{2} \\
& \leq 2\left(\mu_{t}(i)-\alpha_{t}\right)^{2}+4\left(f_{t}(i)-\mu_{t}(i)\right)^{2} .
\end{aligned}
$$

Here, inequality (12) follows because $\left|f_{t}(i)-\mu_{t}(j)\right| \leq 1$ for any $i, j$, and $\left|f_{t}(i)-\alpha_{t}\right| \leq 2$, inequality (13) follows from the fact that $(a+b)^{2} \leq 2 a^{2}+2 b^{2}$ for any real numbers $a, b$, and inequality (14) follows since $16 \eta+16 \eta^{2} \leq 2$ if $\eta \leq 1 / 10$. The lemma follows. 
Proof of Lemma 16 Note that for $t \leq Q, Q_{t}\left(\ell_{t}\right)=\sum_{\tau=1}^{t-1}\left(f_{\tau}(i)-\mu_{\tau}(i)\right)^{2} \leq t$, and for $t>Q$, $Q_{t}\left(\ell_{t}\right) \leq Q$. Thus we have

$$
\sum_{t=1}^{T}\left[\frac{4 \eta}{t} Q_{t}\left(\ell_{t}\right)\right]^{2} \leq 16 \eta^{2} \cdot\left[\sum_{t \leq Q} 1^{2}+\sum_{t>Q} \frac{Q^{2}}{t^{2}}\right] \leq 32 \eta^{2} Q .
$$

Proof of Lemma 17 Let $S=\left\{i: d(i) \leq \frac{\log n}{\eta t}\right\}$, and let $S^{\prime}=I \backslash S$. We upper bound $h\left(\lambda^{I}, d^{I}\right)$ as follows:

$$
\begin{aligned}
h\left(\lambda^{I}, d^{I}\right) & \leq \sum_{i \in S} \frac{\lambda(i) d(i)^{2} \exp (-\eta t d(i))}{\sum_{j \in S} \lambda(j) \exp (-\eta t d(j))}+\sum_{i \in S^{\prime}} \lambda(i) d(i)^{2} \exp (-\eta t d(i)) \\
& \leq \max _{i \in S^{\prime}}\left\{\frac{\lambda(i) d(i)^{2} \exp (-\eta t d(i))}{\lambda(i) \exp (-\eta t d(i))}\right\}+\sum_{i \in S^{\prime}} \frac{\log ^{2} n}{\eta^{2} t^{2}} \exp (-\log n) \\
& \leq \frac{2 \log ^{2} n}{\eta^{2} t^{2}}
\end{aligned}
$$

In (15) we use the inequality $\frac{\sum_{i=1}^{n} a_{i}}{\sum_{i=1}^{n} b_{i}} \leq \max _{i \leq n} \frac{a_{i}}{b_{i}}$ for positive reals $a_{i}$ and $b_{i}$, for bounding the first term. The second term is bounded using the following facts (a) $\lambda(i) \leq 1$, and (b) the function $x^{2} \exp (-\eta t x)$ has a negative derivative (and is thus decreasing) when $x>\frac{2}{\eta t}$, and thus its maximum over the range $\left[\frac{\log n}{\eta t}, 1\right]$ is obtained at $\frac{\log n}{\eta t}$.

\section{Conclusions and future work}

In this paper, we investigated the possibility of bounding the regret of online learning algorithms by terms which depend on the variation of the cost sequence, rather than the number of prediction rounds. We analyzed two algorithms, Lazy Projection and Variation MW, and showed that these algorithms obtain variation-bounded regret. Such bounds are significant not only because they show that it is possible to suffer much less regret than previously believed when the cost sequence is particularly benign, but also because they match the regret bounds of natural regret minimizing algorithms in the stochastic setting of independent cost functions from a fixed distribution.

We believe that this work opens up many new directions for future research, all related to bounding the regret in terms of the variation of the cost sequence in the various different scenarios in which regret minimizing algorithms have been devised: bandit settings, strictly convex cost functions, online convex optimization and so on. We conjecture in all such scenarios, it is possible to get variation-bounded regret. Specifically, we conjecture that any dependence on $T$, the number of prediction rounds, in the regret bound can be replaced by the same dependence on the variation of the cost sequence. Indeed, we have already proved our conjecture true in two more online learning scenarios:

1. Exp-concave cost functions, including the Universal Portfolio Selection problem, for which we obtain $O(\log Q)$ bounds to replace previous bounds of $O(\log T)$ (Hazan and Kale 2009a).

2. Bandit online linear optimization, for which we obtain $O(\sqrt{Q} \cdot \log T)$ bounds to replace previous bounds of $\tilde{O}(\sqrt{T} \cdot \log T)$ (Hazan and Kale 2009b). An open problem is to replace the lower-order term of $O(\log T)$ in the regret bound by $O(\log Q)$. 
In other scenarios, the variation needs to be defined carefully in settings in which it is not natural or obvious, such as in the case of online convex optimization.

Acknowledgements We thank Martin Zinkevich for initial discussions on the possibility of variation bounds on the regret.

\section{References}

Allenberg-Neeman, C., \& Neeman, B. (2004). Full information game with gains and losses. In 15th international conference on algorithmic learning theory.

Auer, P., Cesa-Bianchi, N., Freund, Y., \& Schapire, R. E. (2003). The nonstochastic multiarmed bandit problem. SIAM Journal on Computing, 32(1), 48-77.

Cesa-Bianchi, N., \& Lugosi, G. (2006). Prediction, learning, and games. Cambridge: Cambridge University Press.

Cesa-Bianchi, N., Mansour, Y., \& Stoltz, G. (2007). Improved second-order bounds for prediction with expert advice. Machine Learning, 66(2-3), 21-352.

Cover, T. (1991). Universal portfolios. Mathematical Finance, 1, 1-19.

Freund, Y., \& Schapire, R. E. (1997). A decision-theoretic generalization of on-line learning and an application to boosting. Journal of Computer and System Sciences, 55(1), 119-139.

Hannan, J. (1957). Approximation to Bayes risk in repeated play. In M. Dresher, A. W. Tucker, \& P. Wolfe (Eds.), Contributions to the theory of games (Vol. III, pp. 97-139).

Hazan, E., \& Kale, S. (2009a). On stochastic and worst-case models for investing. In Advances in neural information processing systems (NIPS) (Vol. 22).

Hazan, E., \& Kale, S. (2009b). Better algorithms for benign bandits. In ACM-SIAM symposium on discrete algorithms (SODA09).

Helmbold, D. P., Kivinen, J., \& Warmuth, M. K. (1999). Relative loss bounds for single neurons. IEEE Transactions on Neural Networks, 10(6), 1291-1304.

Herbster, M., \& Warmuth, M. K. (2001). Tracking the best linear predictor. Journal of Machine Learning Research, 1, 281-309.

Kalai, A., \& Vempala, S. (2005). Efficient algorithms for online decision problems. Journal of Computer and System Sciences, 71(3), 291-307.

Kivinen, J., \& Warmuth, M. K. (1997). Exponentiated gradient versus gradient descent for linear predictors. Information and Computation, 132(1), 1-63.

Littlestone, N., \& Warmuth, M. K. (1994). The weighted majority algorithm. Information and Computation, $108(2), 212-261$.

Vovk, V. (1998). A game of prediction with expert advice. Journal of Computer and System Sciences, 56(2), $153-173$.

Zinkevich, M. (2003). Online convex programming and generalized infinitesimal gradient ascent. In ICML (pp. 928-936) 